Acta Crystallographica Section F

Structural Biology

and Crystallization

Communications

ISSN 1744-3091

\section{Stefan Harth, ${ }^{\text {a }}$ Alexander \\ Kotzsch, ${ }^{b}$ Walter Sebald ${ }^{\mathrm{a}}$ and \\ Thomas Dieter Mueller ${ }^{b}$ *}

a Lehrstuhl für Physiologische Chemie II, Biozentrum der Universität Würzburg, Am Hubland, D-97074 Würzburg, Germany, and

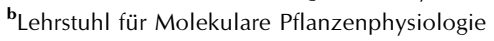
und Biophysik, Julius-von-Sachs Institut der Universität Würzburg, Julius-von-Sachs Platz 2, D-97082 Würzburg, Germany

Correspondence e-mail: mueller@biozentrum.uni-wuerzburg.de

Received 2 June 2010

Accepted 24 June 2010

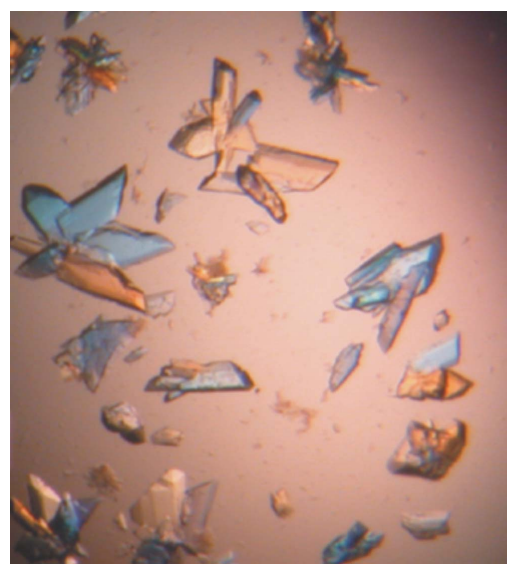

C 2010 International Union of Crystallography All rights reserved

\section{Crystallization of BMP receptor type IA bound to the antibody Fab fragment AbD1556}

An antibody Fab fragment, AbD1556, was selected against the extracellular domain of BMP receptor type IA, which blocks the binding of BMP-2 to BMPRIA and thereby neutralizes BMP-2 activity. To study the mechanism by which BMPR-IA is recognized and bound by the Fab fragment, the complex of AbD1556 bound to BMPR-IA was prepared and crystallized. Crystals of this binary complex belonged to the monoclinic space group $P 2_{1}$, with unit-cell parameters $a=89.32, b=129.25, c=100.24 \AA$ A, $\beta=92.27^{\circ}$.

\section{Introduction}

Bone morphogenetic proteins (BMPs) belong to the large transforming growth factor- $\beta$ (TGF- $\beta$ ) superfamily (Sebald et al., 2004). These secreted cytokines play an important role during early embryonic development as well as in organogenesis (Hogan, 1996; Reddi, 1998). Later, in the adult organism, BMPs and their related analogues exert essential functions in tissue repair and homeostasis, as well as in the control of cell proliferation. Hence, these factors have raised great interest in the fields of regenerative medicine and tissue engineering. Impaired or dysregulated functions of BMPs and other TGF- $\beta$ members have been associated with a large variety of diseases, namely skeletal dysplasias, osteoporosis, fibrosis, infertility and various types of cancer (Gordon \& Blobe, 2008).

At present, more than 40 ligand members of the TGF- $\beta$ superfamily are known and despite their numerous diverse functions the structures of these ligands (as well as the structures of their receptors) are very similar (Sebald et al., 2004). Signalling by BMP ligands requires the binding and heterooligomerization of two types of BMP serine/threonine kinase receptors, termed type I and type II. Upon complex formation of a heterohexameric assembly comprising the dimeric BMP ligand and two type I and two type II receptors, the intracellular kinase domain of the type I receptor is activated by the constitutively active type II receptor kinase, leading to the subsequent activation of SMAD proteins, which themselves migrate to the nucleus and regulate the gene transcription of BMP target genes (Massague, 1998). One hallmark of the TGF- $\beta$ superfamily is the numerical discrepancy between the number of ligands $(\geq 40)$ and the number of available receptors (seven type I and five type II receptors; Sebald et al., 2004). This fact indicates that a TGF- $\beta$ /BMP receptor will usually bind more than one ligand; furthermore, experimental data show that most BMP ligands can also bind to more than one receptor of either subtype. This observation raises two questions: (i) whether and how different TGF- $\beta$ ligands can induce ligand-specific signals despite forming complexes with identical receptor composition and (ii) how the components can recognize and bind various binding partners, which is also known as promiscuity in proteinprotein interaction (Nickel et al., 2009). The BMP receptor type IA is a prime example that is known for its promiscuous binding to different BMP ligands. Structure-function studies of the extracellular domain of BMPR-IA have revealed that BMPR-IA is subject to a 
large conformational change on binding to its ligand BMP-2 (Klages et al., 2008; Kotzsch et al., 2008). The extracellular binding interface of BMPR-IA is largely unfolded in its unbound form, requiring a large induced fit to adopt the conformation when bound to BMP-2. In order to unravel whether the binding promiscuity of BMPR-IA is linked to structural plasticity of its binding interface, we have obtained an antibody Fab fragment against BMPR-IA which blocks binding to BMP-2 and thus occupies an epitope on BMPR-IA that overlaps with that of BMP-2.

\section{Materials and methods}

\subsection{Protein expression and purification}

The extracellular domain of human BMPR-IA (BMPR-IA EC; $_{\text {; }}$ residues 1-129 of the mature part; Swiss-Prot entry P36894) was fused C-terminally to thioredoxin (Trx) behind a $\mathrm{His}_{6}$ tag followed by a thrombin cleavage site. This construct was expressed in the cytoplasm of Escherichia coli strain AD494 (DE3) following the protocol of Kirsch et al. (2000) with modifications. These modifications applied to the purified Trx-BMPR-IA fusion protein after cell lysis and initial $\mathrm{Ni}^{2+}$ metal-affinity chromatography. After proteolysis of the purified Trx-BMPR-IA fusion protein using $0.3 \mathrm{U}$ thrombin per milligram of Trx-BMPR-IA $\mathrm{EC}_{\mathrm{EC}}$ for $5 \mathrm{~h}$ at $303 \mathrm{~K}$, the protein solution containing His $_{6}$-tagged thioredoxin and BMPR-IA $\mathrm{EC}_{\text {(carrying an additional }}$ Gly-Ser motif at the N-terminus resulting from the thrombin cleavage) was incubated for $72-96 \mathrm{~h}$ at $277 \mathrm{~K}$ to maximize the refolding of the BMPR-IA $\mathrm{EC}_{\mathrm{C}}$ protein, providing a fourfold to fivefold increase in active BMPR-IA protein. His-tagged thioredoxin was separated from monomeric and multimeric BMPR-IA $\mathrm{EC}_{\mathrm{EC}}$ by anion-exchange chromatography using TMAE resin and employing a linear gradient of 0 $1 M \mathrm{NaCl}$ in $20 \mathrm{~m} M$ Tris $\mathrm{pH} \mathrm{8.0,} \mathrm{with} \mathrm{thioredoxin} \mathrm{eluting} \mathrm{first} \mathrm{at}$ $75 \mathrm{mM} \mathrm{NaCl}$ and monomeric as well as multimeric forms of BMPRIA $_{\mathrm{EC}}$ eluting at $150 \mathrm{mM} \mathrm{NaCl}$. Active monomeric BMPR-IA $\mathrm{EC}_{\mathrm{EC}}$ was then obtained by a final affinity-chromatography step using a BMP-2 affinity matrix as described by Kirsch et al. (2000).

Antibody Fab fragments selected against the extracellular domain of BMPR-IA were obtained from AbD Serotec (Morphosys Inc.) in a format containing a noncleavable Strep-tag (peptide sequence SAWHPQFEK) at the C-terminus of the heavy chain and were used without further purification.

\subsection{Preparation and crystallization of the Fab-BMPR-IA complexes}

To obtain a homogenous antibody-receptor protein complex, the Fab AbD1556 was mixed with a $10 \%$ molar excess of BMPR-IA $\mathrm{EC}_{\mathrm{EC}}$ in $10 \mathrm{~m} M$ HEPES pH 7.4, $150 \mathrm{~m} M \mathrm{NaCl}$ and incubated for $30 \mathrm{~min}$. The protein solution was concentrated to $10 \mathrm{mg} \mathrm{ml}^{-1}$ using ultrafiltration and excess BMPR-IA was removed by subsequent gel filtration using a Superdex 200 HR 30/10 column with $10 \mathrm{~m} M$ HEPES pH 7.4, $150 \mathrm{~m} M \mathrm{NaCl}$ as the running buffer. Fractions that contained $\mathrm{Fab}$ AbD1556 and BMPR-IA $\mathrm{EC}_{\mathrm{EC}}$ in an equimolar ratio were pooled and the protein solution was concentrated to $16 \mathrm{mg} \mathrm{ml}^{-1}$ via ultrafiltration for crystallization. Initial screening for crystallization of the FabBMPR-IA complex was performed using commercially available sparse-matrix screens, namely Index, PEG/Ion and SaltRx from Hampton Research. In addition, we used a screen developed in our laboratory based on a compilation of crystallization conditions that have successfully been employed in the crystallization of various antibody-antigen complexes. Crystallization trials were performed using a sitting-drop vapour-diffusion setup and the crystals used for data acquisition were obtained from hanging-drop vapour-diffusion experiments. In all crystallization setups $1 \mu \mathrm{l}$ protein solution was mixed with $1 \mu \mathrm{l}$ reservoir solution in the droplet. Successful crystallization conditions for the AbD1556-BMPR-IA EC $_{\text {complex usually }}$ contained polyethylene glycols with a molecular weight of between 3350 and 12000 as a precipitant and buffers that maintain a $\mathrm{pH}$ between 6.5 and 8.0. From optimization of the PEG species, precipitant concentration and $\mathrm{pH}$, we obtained a final crystallization condition for AbD1556-BMPR-IA EC $_{\text {C }}$ consisting of $20 \%(w / v)$ PEG $8000,0.1 M$ Tris- $\mathrm{HCl}$ pH 7.0 with $10-12 \%(w / v)$ glucose as a cryoprotectant, from which crystals grew to dimensions of about $150 \times$ $150 \times 40 \mu \mathrm{m}$ at $294 \mathrm{~K}$ within $7 \mathrm{~d}$.

\subsection{Data collection}

A complete data set for the AbD1556-BMPR-IA EC $_{\text {complex was }}$ acquired from a single crystal using a home X-ray source (Rigaku MicroMax-007 X-ray generator, VarixMax HR mirror optics and a Rigaku R-AXIS IV++ detector). Crystals of AbD1556-BMPR-IA EC harvested from crystallization solution composed of $20 \%(w / v)$ PEG $8000,0.1 M$ Tris- $\mathrm{HCl} \mathrm{pH} 7.0$ and $12 \%(w / v)$ glucose (as a cryoprotectant) were directly flash-frozen in liquid nitrogen. The crystal-todetector distance was set to $130 \mathrm{~mm}$ and data were collected at $100 \mathrm{~K}$ by rotating the crystal through $162.5^{\circ}\left(0.5^{\circ}\right.$ oscillation $)$ with $200 \mathrm{~s}$ exposure per frame. The data were indexed, integrated and scaled using CrystalClear v.1.3.6 SP1 (Rigaku).

\section{Results and discussion}

\subsection{Crystallization of the Fab-BMPR-IA ectodomain complexes}

Structural analyses of different BMP ligand-receptor complexes have raised the question of whether the inherent structural flexibility and plasticity in the complex components provides the molecular mechanism for the pronounced ligand-receptor promiscuity that is a hallmark of the TGF- $\beta$ /BMP superfamily (Nickel et al., 2009). A structure of the extracellular domain of BMPR-IA recently determined by NMR spectroscopy further fuelled this hypothesis, showing that a major part of the ligand-binding epitope of BMPR-IA is unfolded and dynamic prior to ligand binding (Klages et al., 2008). Specifically, the short $\alpha$-helical element in the $\beta 4-\beta 5$ loop of BMPRIA, which is in the centre of the epitope and carries the main binding determinants for the ligand-receptor interaction (Keller et al., 2004), is absent in the free form of BMPR-IA. Further investigations into the conformational rearrangements of BMPR-IA led to the hypothesis that this $\alpha$-helix exists in a so-called statu nascendi. Thus, the helix element forms spontaneously upon changes in the environment or complex formation. To test this hypothesis, we attempted to determine the structure of BMPR-IA bound to different binding partners. In collaboration with AbD Serotec, Fab fragments were selected against the extracellular domain of BMPR-IA employing phage display. For selection of the Fab antibodies, the HuCal antibody library of MorphoSys was used (Knappik et al., 2000; Rothe et al., 2008), BMPR-IA $\mathrm{EC}_{\mathrm{EC}}$ was biotinylated using Sulfo-NHS-LC-biotin (Pierce) according to the manufacturer's recommendations and 'antigen'-binding Fab-presenting phages were selected by solution panning (Steidl et al., 2008). After several rounds of selection, Fab fragments were screened by DNA sequencing and unique DNA sequences were cloned into an expression vector, allowing high-level expression in E. coli. The Fab antibodies used in this study carry a 10-mer Strep-tag at the C-terminus of the heavy chain which was not removed for crystallization. Eight different Fabs against BMPR-IA $\mathrm{EC}_{\mathrm{C}}$ were tested, of which two, AbD1556 and AbD1564, exhibited neutralizing activity, i.e. they were able to block the binding of BMP-2 to BMPR-IA, thereby neutralizing BMP-2 signalling in alkaline 
Table 1

Data-collection and processing statistics for the native crystal of AbD1556-BMPR$\mathrm{IA}_{\mathrm{EC}}$.

Values in parentheses are for the highest resolution shell.

\begin{tabular}{ll}
\hline Beamline & Home source \\
Wavelength $(\AA)$ & 1.5418 \\
Temperature $(\mathrm{K})$ & 100 \\
Space group & $P 2_{1}$ \\
Unit-cell parameters $\left.\left(\AA{ }^{\circ}\right)^{\circ}\right)$ & $a=89.32, b=129.25$, \\
& $c=100.24, \beta=92.27$ \\
Resolution $(\AA)$ & $30.4-2.70(2.80-2.70)$ \\
No. of reflections collected & $155123(15796)$ \\
No. of unique reflections & $59539(6237)$ \\
Completeness $(\%)$ & $95.3(97.8)$ \\
Multiplicity & $2.6(2.6)$ \\
$R_{\text {merge }} \dagger$ & $0.08(0.341)$ \\
$\langle I / \sigma(I)\rangle$ & $8.2(2.7)$ \\
$\chi^{2}$ & $0.98(1.19)$ \\
\hline
\end{tabular}

$\dagger R_{\text {merge }}=\sum_{h k l} \sum_{i}\left|I_{i}(h k l)-\langle I(h k l)\rangle\right| / \sum_{h k l} \sum_{i} I_{i}(h k l)$, where $I_{i}(h k l)$ is the intensity of the $i$ th observation of the unique reflection $h k l$ and $\langle I(h k l)\rangle$ is the mean of the intensities of all observations of reflection $\mathrm{hkl}$.

phosphatase expression (ALP) assays. Owing to their BMP-2blocking nature AbD1556 and AbD1564 should have overlapping binding epitopes with BMP-2 and are thus ideally suited for studying the influence of different binding partners on the flexibility of the BMPR-IA binding epitope.

Binary complexes of AbD1556 or AbD1564 bound to BMPR-IA $A_{E C}$ were prepared by mixing antibody protein and BMPR-IA $\mathrm{EC}_{\mathrm{EC}}$ in a 1:1.1 ratio and removing excess receptor or Fab protein by subsequent gel filtration. Fractions containing either Fab-receptor complex were pooled, concentrated to $16 \mathrm{mg} \mathrm{ml}^{-1}$ by ultrafiltration and subjected to crystallization using various commercially available crystallization screening kits and vapour-diffusion techniques. For the AbD1564BMPR-IA $A_{E C}$ complex crystals could be obtained from two different conditions, but the crystals obtained using either condition only diffracted to very low resolution ( $\geq 7 \AA$ ). Despite extensive optimization screening, the diffraction properties of these crystals could not be improved and thus focus was directed towards crystallization of

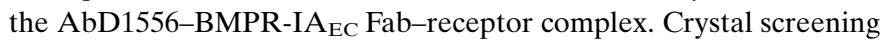
of this complex yielded several successful conditions from the Index, PEG/Ion and SaltRX kits. In addition, we also performed crystallization screening using a home-made sparse-matrix screen compiled

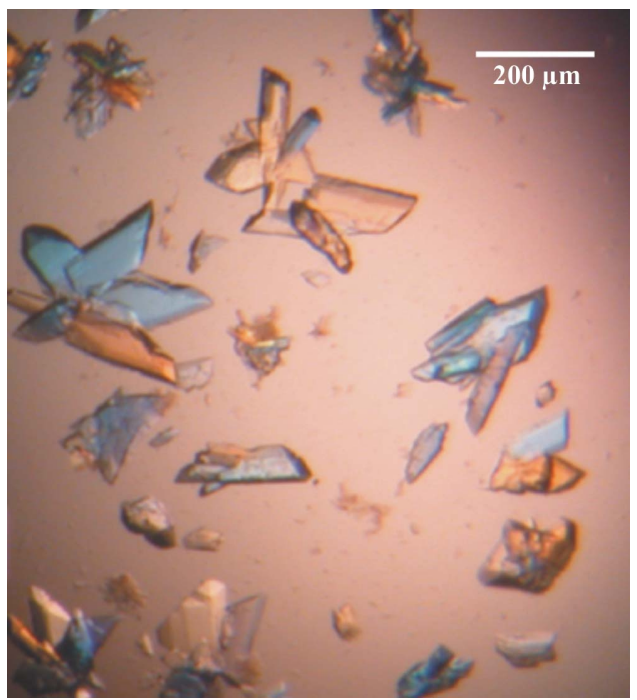

Figure 1

Crystals of the AbD1556-BMPR-IA $\mathrm{EC}_{\mathrm{EC}}$ complex. The crystals grew to maximum dimensions of $200 \times 50 \times 20 \mu \mathrm{m}$ within $7 \mathrm{~d}$. from conditions reported for antibody-antigen complexes in the PDB. Several conditions that led to the growth of large crystals of AbD1556-BMPR-IA $\mathrm{EC}_{\mathrm{EC}}$ were obtained, all of which were based on the use of polyethylene glycols of molecular weight between 3350 and 12000 as a precipitant. Optimization screening finally yielded an optimal crystallization condition composed of 20\%(w/v) PEG 8000, $0.1 M$ Tris- $\mathrm{HCl} \mathrm{pH} 7.0$ and $12 \%$ glucose (as a cryoprotectant), from which large crystals of the complex could be grown at $294 \mathrm{~K}$ within one week (Fig. 1). SDS-PAGE analysis of these crystals confirmed the presence of the antibody-antigen complex.

\subsection{Data acquisition and preliminary analysis}

Although the crystals of the AbD1556-BMPR-IA EC $_{\text {complex had }}$ a tendency to grow as stacks, large single crystals could easily be obtained by careful manual trimming (Fig. 1). Such crystals of the AbD1556-BMPR-IA EC complex diffracted to $2.6 \AA$ resolution and belonged to space group $P 2_{1}$, with unit-cell parameters $a=89.32$, $b=129.25, c=100.24 \AA, \beta=92.3^{\circ}$. A complete native data set with a maximum resolution of $2.7 \AA$ was acquired using an X-ray home source (Fig. 2). The data set, comprising 325 frames with $0.5^{\circ}$ oscillation, consisted of 155123 reflections, of which 59539 were unique. The overall $R_{\text {merge }}$ was $8.0 \%$ in the resolution range $30.4-2.7 \AA$ and the completeness was $95.3 \%$ (Table 1). Calculation of the Matthews coefficient (Matthews, 1968) suggested the presence of either three $\left(60 \%\right.$ solvent content, $\left.V_{\mathrm{M}}=3.06 \AA^{3} \mathrm{Da}^{-1}\right)$ or four $(46 \%$ solvent content, $V_{\mathrm{M}}=2.29 \AA^{3} \mathrm{Da}^{-1}$ ) AbD1556-BMPR-IA $\mathrm{EC}$ complexes in the asymmetric unit. Since SDS-PAGE analysis of the crystals clearly suggested a 1:1 ratio of $\mathrm{Fab}$ and receptor protein in the crystals, a different packing with free and BMPR-IA $\mathrm{EC}_{\text {-bound AbD1556 }}$ seemed unlikely. To resolve the presence of noncrystallographic symmetry, the software packages phenix.xtriage (Adams et al., 2010) and GLRF (Tong \& Rossmann, 1997) were used to check for the presence of pseudotranslation and NCS symmetry. While pseudotranslation could not be detected from analysis of the native

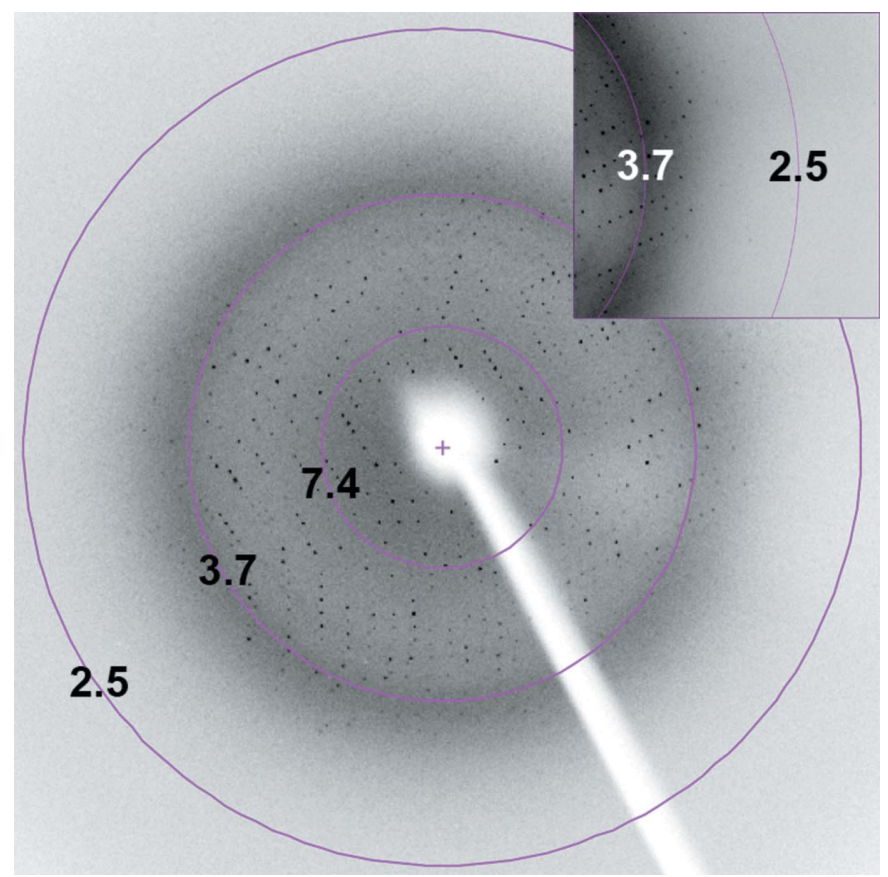

Figure 2

Diffraction pattern and diffraction limit of a crystal from Fig. 1. The diffraction limit of these crystals was about $2.6 \AA$. The resolution limits (in $\AA$ ) are indicated by magenta circles. 
Patterson map using phenix.xtriage (the largest non-origin peaks in the Patterson were less than $5 \%$ of the origin peak), two twofold axes could be detected from a self-rotation calculation using GLRF on the $\kappa=180^{\circ}$ section, which were perpendicular to each other and were oriented at about $45^{\circ}$ with respect to the crystallographic twofold (Fig. 3). Considering the additional absence of any peaks in the $\kappa=120^{\circ}$ section, the crystal is very likely to contain four AbD1556BMPR-IA $\mathrm{EC}_{\mathrm{EC}}$ complexes in the asymmetric unit. To solve the structure of the complex, molecular replacement was employed using the software packages CNS (Brunger, 2007) and Phaser (McCoy et al., 2007). The Fab fragment with high affinity for the tetanus toxoid (PDB code 1aqk; Faber et al., 1998) was selected as the search template for the Fab fragment on the basis of its highest overall sequence similarity to the AbD1556 Fab. The structure of BMPRIA $\mathrm{EC}_{\mathrm{EC}}$ bound to BMP-2 (PDB code 1rew; Keller et al., 2004) was used as a search template for the receptor ectodomain. Owing to the number of complexes in the asymmetric unit, the first rotationfunction and translation-function searches were performed using the structure of the complete Fab fragment (1aqk template) employing the software package CNS (resolution range 30-4 $\mathrm{A}$ ), but no clear solution could be obtained. One possible reason for the failure to find a molecular-replacement solution might be the variable hinge angle between the constant and variable region of the antibody.

Therefore, the Phaser software was used for subsequent molecular replacement owing to its capability to search consecutively with fragments of the search template. In the first run, the Fab template 1aqk was dissected into four substructures comprising the constant and variable regions of the light and heavy chains as individual domains. Using these search templates, we obtained a first solution showing that the packing between the variable regions of the light and heavy chains as well as between the constant regions of the light and heavy chains are identical to the full template 1aqk (Table 2). Run $A$ in Table 2 shows an example of a search using only the constant region of the Fab 1aqk. This observation suggests that the substructures for the variable and the constant region are conserved in the structure of AbD1556 and only the interdomain hinge angle

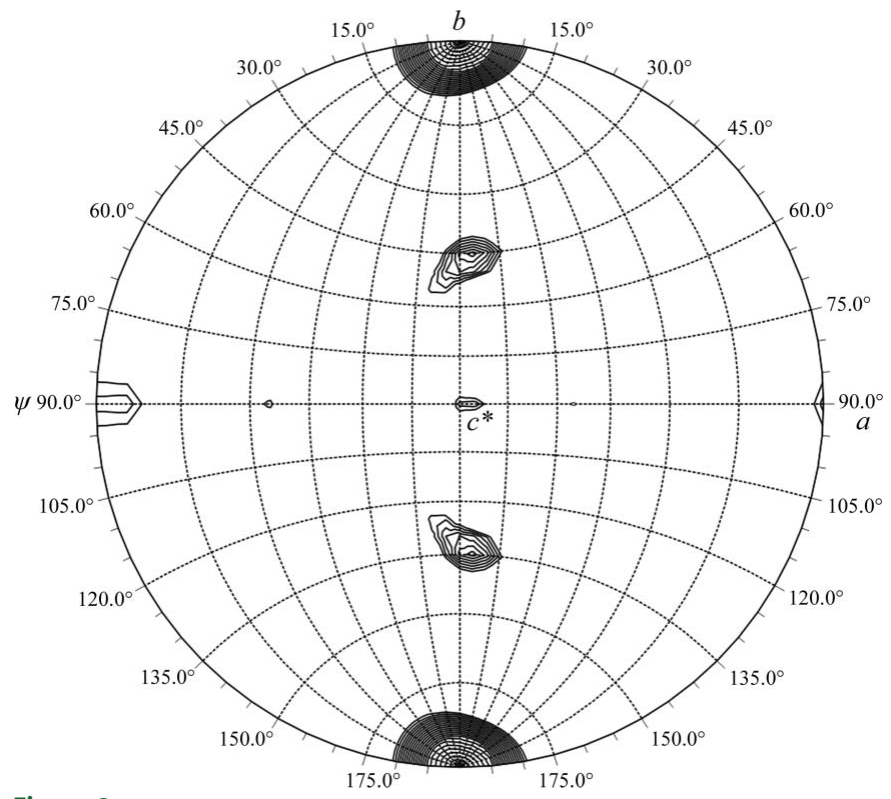

Figure 3

Section of a self-rotation calculation using the software $G L R F$ at $\kappa=180^{\circ}$. In addition to the crystallographic twofold observed at the top and bottom of the plot, two noncrystallographic twofolds that are perpendicular to each other and share an angle of about $45^{\circ}$ with the crystallographic twofold axes are seen.
Table 2

Molecular-replacement (MR) solutions for the AbD1556-BMPR-IA $\mathrm{EC}_{\mathrm{EC}}$ complex obtained with Phaser.

All MR runs using Phaser were performed using resolution limits of 30-2.7 $\AA$. The similarity of the template was set to 0.7 , four search templates were assumed to be present in the asymmetric unit and the packing tolerance was set to a maximum of ten clashes.

\begin{tabular}{|c|c|c|c|}
\hline & $\mathrm{RFZ} \dagger$ & TFZ末 & LLG§ \\
\hline \multicolumn{4}{|l|}{ Run $A$} \\
\hline 1. Constant domain & 5.5 & 7.1 & 103 \\
\hline 2. Constant domain & 6.2 & 15.0 & 241 \\
\hline 3. Constant domain & 6.2 & 15.0 & 241 \\
\hline 4. Constant domain & 5.7 & 21.6 & 127 \\
\hline \multicolumn{4}{|l|}{ Run $B$} \\
\hline 1. Fab†† & 14.6 & 16.8 & 400 \\
\hline 2. Fab & 14.2 & 38.6 & 1417 \\
\hline 3. Fab & 13.9 & 44.4 & 2798 \\
\hline 4. Fab & 11.6 & 46.1 & 4110 \\
\hline 1. BMPR-IA $+\$$ & 13.4 & 16.7 & 4288 \\
\hline 2. BMPR-IA & 3.9 & 10.9 & 4458 \\
\hline \multicolumn{4}{|l|}{ Run $C$} \\
\hline 1. Fab-BMPR-IA complex $\S$ & 15.4 & 18.2 & 429 \\
\hline 2. Fab-BMPR-IA complex & 16.4 & 41.3 & 1631 \\
\hline 3. Fab-BMPR-IA complex & 12.4 & 43.8 & 3278 \\
\hline 4. Fab-BMPR-IA complex & 12.7 & 52.3 & 5318 \\
\hline
\end{tabular}

$\dagger$ Rotation-function $Z$ score. $\quad$ 末 Translation-function $Z$ score. $\$$ Log-likelihood gain. For judgement of a correct MR solution, TFZ should be higher than 5 and for consecutive seaches of substructures LLG should be positive and increase with the addition of correctly placed search templates. Constant domain: only the constant domains of the heavy and light chains of the Fab search template 1aqk (residues Ser125-Cys226 of the heavy chain and Pro113-Ser216 of the light chain) were used. $\dagger \dagger$ A full Fab reconstructed from the searches using only the variable or constant regions comprising residues Val2-Val121 and Ser125-Cys226 of the heavy chain and Asn2-Gly111 and Pro113-Ser216 of the light chain were used. +5 The coordinates of the BMPR-IA of the complex BMP-2-BMPR-IA $\mathrm{EC}_{\mathrm{EC}}$ (PDB entry 1rew) were used as the search template. $\S \S$ A full Fab-BMPR-IA $\mathrm{EC}_{\mathrm{E}}$ complex comprising the coordinates of the reconstructed Fab template used in Run $B$ and the bound BMPR-IA $\mathrm{EC}_{\mathrm{EC}}$ as observed in the MR solution in Run $B$ was used in the final search

between the variable and constant region within a chain differs between the Fab template 1aqk and the Fab AbD1556. Therefore, the variable and constant regions of the original Fab against tetanus toxoid (1aqk) were used as individual search templates together with the structure of BMPR-IA. Phaser was used to search for four substructures resembling four Fab fragments and several BMPR-IA molecules in the asymmetric unit. The solution of the latter search allowed the reconstruction of the Fab fragment with an adjusted hinge angle and reflecting the constant/variable-region architecture present in the Fab AbD1556. A clear molecular-replacement solution (Table 2, Run $B$ ) could then be obtained for the placement of four

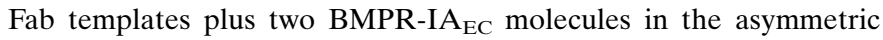
unit. Searching for additional BMPR-IA molecules resulted in too many clashes in the packing of the molecules in the asymmetric unit; increasing the packing tolerance in Phaser led to the misplacement of these additional BMPR-IA molecules. However, inspection of initial electron-density maps clearly showed that in accordance with the SDS-PAGE analysis all four Fab molecules in the asymmetric unit are bound to a BMPR-IA moiety. Thus, a final search using a full Fab 1aqk-BMPR-IA $\mathrm{EC}_{\mathrm{C}}$ complex as found in the earlier molecularreplacement run (Table 2, Run $B$ ) as a template finally succeeded in finding all four Fab-BMPR-IA complexes in the asymmetric unit (Table 2, Run $C$ ). Initial density-map calculations show all parts of the Fab search template-BMPR-IA ${ }_{\mathrm{EC}}$ complex to be covered by electron density; however, steric clashes between receptor and Fab molecules show that structures of the components still require refinement to yield proper packing. The structure of the Fab AbD1556-BMPR$\mathrm{IA}_{\mathrm{EC}}$ complex is currently being refined; the sequences of the Fab search template are being exchanged for the sequence of AbD1556 and conformational differences in the Fab CDR loops as well as in BMPR-IA $A_{E C}$ are being modelled. The final structure of BMPR-IA $\mathrm{EC}_{\mathrm{C}}$ 


\section{crystallization communications}

bound to the neutralizing Fab AbD1556 is likely to provide further insight into the structural plasticity of the BMPR-IA binding epitope.

We would like to thank Achim Knappik from AbD Serotec for his help and efforts in obtaining the Fab antibodies directed against BMPR-IA. The authors also thank Werner Schmitz for his support in mass-spectrometric analyses. We gratefully acknowledge access to the $\mathrm{X}$-ray facility at the Rudolf Virchow Center of the University of Würzburg. This project was supported by the Deutsche Forschungsgemeinschaft (DFG), SFB 487 TP B1 and B2.

\section{References}

Adams, P. D. et al. (2010). Acta Cryst. D66, 213-221.

Brunger, A. T. (2007). Nature Protoc. 2, 2728-2733.

Faber, C., Shan, L., Fan, Z., Guddat, L. W., Furebring, C., Ohlin, M., Borrebaeck, C. A. \& Edmundson, A. B. (1998). Immunotechnology, 3, 253-270.

Gordon, K. J. \& Blobe, G. C. (2008). Biochim. Biophys. Acta, 1782, 197-228. Hogan, B. L. (1996). Curr. Opin. Genet. Dev. 6, 432-438.
Keller, S., Nickel, J., Zhang, J.-L., Sebald, W. \& Mueller, T. D. (2004). Nature Struct. Mol. Biol. 11, 481-488.

Kirsch, T., Nickel, J. \& Sebald, W. (2000). FEBS Lett. 468, 215-219.

Klages, J., Kotzsch, A., Coles, M., Sebald, W., Nickel, J., Müller, T. \& Kessler, H. (2008). Biochemistry, 47, 11930-11939.

Knappik, A., Ge, L., Honegger, A., Pack, P., Fischer, M., Wellnhofer, G., Hoess, A., Wölle, J., Plückthun, A. \& Virnekäs, B. (2000). J. Mol. Biol. 296, $57-86$.

Kotzsch, A., Nickel, J., Seher, A., Heinecke, K., van Geersdaele, L., Herrmann, T., Sebald, W. \& Mueller, T. D. (2008). J. Biol. Chem. 283, 5876-5887.

Massague, J. (1998). Annu. Rev. Biochem. 67, 753-791.

Matthews, B. W. (1968). J. Mol. Biol. 33, 491-497.

McCoy, A. J., Grosse-Kunstleve, R. W., Adams, P. D., Winn, M. D., Storoni, L. C. \& Read, R. J. (2007). J. Appl. Cryst. 40, 658-674.

Nickel, J., Sebald, W., Groppe, J. C. \& Mueller, T. D. (2009). Cytokine Growth Factor Rev. 20, 367-377.

Reddi, A. H. (1998). Nature Biotechnol. 16, 247-252.

Rothe, C. et al. (2008). J. Mol. Biol. 376, 1182-1200.

Sebald, W., Nickel, J., Zhang, J.-L. \& Mueller, T. D. (2004). Biol. Chem. 385, 697-710.

Steidl, S., Ratsch, O., Brocks, B., Durr, M. \& Thomassen-Wolf, E. (2008). Mol. Immunol. 46, 135-144.

Tong, L. \& Rossmann, M. G. (1997). Methods Enzymol. 276, 594-611. 